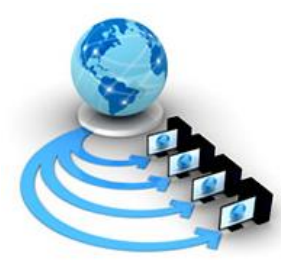

Volume 9, No. 3, May-June 2018

International Journal of Advanced Research in Computer Science

RESEARCH PAPER

Available Online at www.ijarcs.info

\title{
SOME FIXED POINT THEOREMS IN CONE RANDOM METRIC SPACE USING RANDOM OPERATORS
}

\author{
Gourish Parashar \\ Department of Mathematics TIT \& \\ Science Bhopal and Research Scholar \\ M.G.C.G.V. Chitrakoot (M.P.), India \\ Manoj Kumar Shukla \\ Department of Mathematics, \\ Institute for Excellence in Higher Education, \\ Bhopal, (M.P.), India
}

\author{
Anil Agrawal \\ Department of Mathematics \\ M.G.C.G.V. Chitrakoot (M.P.), India
}

\begin{abstract}
Some fixed point theorems in cone random metric space by use of random operators with different contractions are proved which are generalizations of contraction mappings considered by various researchers.
\end{abstract}

Keyword: Cone Random Metric Space, Random operator, Random fixed point, Implicit relation.

AMS CLASSIFICATION: 47H10, 54H25.

\section{INTRODUCTION}

Random fixed point theorems for contraction mappings in polish spaces and random fixed point theorems are of fundamental importance in probabilistic functional analysis. The Random fixed point theorems were initially studied by the Prague school of Probabilistic. In 1955 it was Spacek [28] who introduced this topic further Hans[11], [12], extended the work of Spacek. Subsequently Kannan, R. [16], Bharucha-Ried [8], Itoh [15] proved several random fixed point theorems and gave their applications to random differential equations in Banach spaces. Random coincidence point theorems and random fixed point theorem are stochastic generalization of classical coincidence point theorems and classical fixed point theorems. Sehgal and Singh [26], Papageorgiou [22], Rhoades Sessa Khan [25] and Lin [19] have proved differential stochastic version of well known Schauder's fixed point theorem. Then Beg, I. and Azam [2], Beg and Shahzad [3], [4], [5], studied the structure of common random fixed points and random coincidence points of a pair of compatible random operators.

Further the cone metric space was defined by Huang and Zhang [14], they generalized the concept of metric spaces, replacing the set of real numbers by an ordered Banach space; Huang and Zhang also described the convergence of sequences and introduced the notion of completeness in cone metric spaces. They have proved some fixed point theorems of contractive mappings on complete cone metric space with the assumption of normality of a cone. There exist a lot of work involving fixed point used the Banach contraction principle. This principle has been extended kind of contraction mappings considered by various authors[1],[6],[7],[9], [13] and [27]. Recently, in 2008 various authors such as Dhagat, V. B, Sharma, A. K, Bhardwaj, R. K. [10], have studied Fixed point theorem for
Random operators in Hilbert Spaces, this result was a generalization of the results of Huang and Zhang also they studied fixed point theorems for normal and non-normal cones. Recently Rezapour et.al.[23] ,[24] Pathak, H. K. and Shahzad, N.[17] did some notable work in quashi contraction maps and cone metric spaces with contractive mapping. Then in 2010 Sumitra et. al. [29] and Mehta Smrati [20] in 2011 extended the previous results in different spaces. Here, we have prove some fixed point theorems in cone random metric space by using random operators with different contractions.

\section{PRELIMINARIES}

Definition 2.1: Let $(E, \tau)$ be a topological vector space and $P$ a subset of $E, P$ is called a cone if

1. $P$ is non-empty and closed, $P \neq\{0\}$,

2. For $x, y \in P$ and $a, b \in R \Rightarrow a x+b y \in P$ where $a, b \geq 0$

3. If $x \in P$ and $-x \in P \Rightarrow x=0$

For a given cone $P \subseteq E$, a partial ordering $\leq$ with respect to $P$ is defined by $x \leq y$ if and only if $y-x \in P, x<y$ if $x \leq$ $y$ and $x \neq y$,while $x \ll y$ will stand for $y-x \in$ int $P$ denotes the interior of $P$.

Definition 2.2: Measurable function: Let $(\Omega, \Sigma)$ be a measurable space with $\sum$ a sigma algebra of subsets of $\Omega$ and $M$ a non-empty subset of a metric space $X=(X, d)$. Let $2^{M}$ be the family of all non-empty subsets of $M$ where $C(M)$ the family of all nonempty closed subsets of $M$. A mapping $G: \Omega \rightarrow 2^{M}$ is called measurable if, for each open subset $U$ of $M$, such that 
$G^{-1}(U) \in \sum, \quad$ where $\quad G^{-1}(U)=$

$\{\omega \in \Omega: G(\omega) \cap U \neq \emptyset\}$.

Definition 2.3: Measurable selector [18]: A mapping $\xi$ : $\Omega \rightarrow M$ is called a measurable selector of a measurable mapping $G: \Omega \rightarrow 2^{M}$ if $\xi$ is measurable and $\xi(\omega) \in$ $G(\omega)$ for each $\omega \in \Omega$.

Definition 2.4: Random operator [21]: Mapping $T: \Omega \times$ $M \rightarrow X$ is said to be a random operator if, for each fixed $x \in$ $M, T(., x): \Omega \rightarrow X$ is measurable.

Definition 2.5: Continuous Random operator [21]: A random operator $T: \Omega \times M \rightarrow X$ is said to be continuous random operator if, for each fixed $x \in M, T(., x): \Omega \rightarrow X$ is continuous.

Definition 2.6: Random fixed point: A measurable mapping $\xi: \Omega \rightarrow M$ is a random fixed point of a random operator $T: \Omega \times M \rightarrow X$ if $\xi(\omega)=T(\omega, \xi(\omega))$ for each $\omega \in \Omega$.

Definition 2.7: Let $M$ be a nonempty set and the mapping $d: \Omega \times M \rightarrow X$ and Pᄃ $X$ be a cone, $\omega \in \Omega$ be a selector, satisfies the following conditions:

2.7(a) $d(x(\omega), y(\omega))>0 \forall x(\omega), y(\omega) \in \Omega \times X \Leftrightarrow$ $x(\omega)=y(\omega)$

2.7(b) $\quad d(x(\omega), y(\omega))=d(y(\omega), x(\omega)) \forall x, y \in$ $X, \omega \in \Omega$ and $x(\omega), y(\omega) \in \Omega \times X$

2.7(c) $d(x(\omega), y(\omega))=d(x(\omega), z(\omega))+$ $d(z(\omega), y(\omega)) \forall x, y \in X$ and $\omega \in \Omega$ be a selector.

2.7(d) for any $x, y \in X, \omega \in \Omega$,

$d(x(\omega), y(\omega))$ is non-increasing and left continuous in $\alpha$.

Then $d$ is called cone random metric in $M$ and $(M, d)$ is a cone random metric space.

\section{Definition 2.8: Implicit Relation}

Let $\Phi$ be the class of all real-valued continuous functions : $\left(R^{+}\right)^{3} \rightarrow R^{+}$non-decreasing in the first argument and satisfying the following conditions:

$x \leq \varphi(y, x+y, y)$ or $x \leq \varphi(y, x, x)$ or $x \leq \varphi(y, x+$ $y, x+y)$

there exists a real number $0<h<1$ such that $x \leq h y$, for all $x, y \geq 0$.

Similarly for $\left(R^{+}\right)^{5}$, Let $\Phi$ be the class of all real-valued continuous functions $\varphi:\left(R^{+}\right)^{5} \rightarrow R^{+}$non-decreasing in the first argument and satisfying the following conditions for all $x, y \geq 0$.

$\boldsymbol{x} \leq \varphi(y, x+y, x+y, x+y, y)$ or $\boldsymbol{x} \leq \varphi(y, 0, y, y, x+y)$

or $\boldsymbol{x} \leq \varphi(y, y, x, y, x)$

there exists a real number $0<h<1$ such that $x \leq h y$.

\section{MAIN RESULTS}

Theorem 3.1: Let $(X, d)$ be a complete cone random metric space and let $M$ be a non-empty separable closed subset of cone metric space $X$ and let $T$ be a continuous random on $M$ such that for all $\omega \in \Omega, T(\omega,):. \Omega \times M \rightarrow M$ satisfying contraction

$d(T(x(\omega)), T(y(\omega))) \leq$

$\phi(d(x(\omega), y(\omega)), d(y(\omega), T(x(\omega))+d(y(\omega), T(y(\omega))$,

$d(x(\omega), T(x(\omega))+d(y(\omega), T(x(\omega))$,

$d(x(\omega), T(y(\omega), T(y(\omega), T(x(\omega))))$

For all, $y \in X, \omega \in \Omega$. Then $T$ has a fixed point in $X$.
Proof: For each $x_{0}(\omega) \in \Omega \times X$ and $n \geq 1$, let $x_{1}=T x_{0}$ And $x_{n+1}(\omega)=T\left(x_{n}(\omega)\right)=T^{n+1} x_{0}(\omega)$. Then $\left.d\left(x_{n}(\omega), x_{n+1}(\omega)\right)=d\left(x_{n-1}(\omega)\right), T\left(x_{n}(\omega)\right)\right)$ $\leq \phi\left(d\left(x_{n-1}(\omega), x_{n}(\omega)\right), d\left(x_{n}(\omega), T\left(x_{n-1}(\omega)\right)+\right.\right.$ $d\left(x_{n}(\omega), T\left(x_{n}(\omega)\right)\right.$,

$d\left(x_{n-1}(\omega), T\left(x_{n-1}(\omega)\right)+d\left(x_{n}(\omega), T\left(x_{n-1}(\omega)\right)\right.\right.$

$d\left(x_{n-1}(\omega), T\left(x_{n}(\omega)\right)+d\left(x_{n}(\omega), T\left(x_{n-1}(\omega)\right)\right)\right.$

$\leq$

$\emptyset\left(d\left(x_{n-1}(\omega), x_{n}(\omega)\right), d\left(x_{n}(\omega), x_{n}(\omega)\right), d\left(x_{n}(\omega), x_{n+1}(\omega)\right)\right.$,

$d\left(x_{n}(\omega), x_{n}(\omega)\right)+d\left(x_{n-1}(\omega), x_{n}(\omega)\right)$,

$\left.d\left(x_{n-1}(\omega), x_{n+1}(\omega)\right)+d\left(x_{n}(\omega), x_{n}(\omega)\right)\right)$

$\leq \phi\left(d\left(x_{n-1}(\omega), x_{n}(\omega)\right), 0+\right.$

$d\left(x_{n}(\omega), x_{n+1}(\omega)\right), d\left(x_{n-1}(\omega), x_{n}(\omega)\right)+0$,

$\left.d\left(x_{n-1}(\omega), x_{n}(\omega)\right)+d\left(x_{n}(\omega), x_{n+1}(\omega)\right)+0\right)$

Therefore by definition (2.8) we have

$d\left(x_{n}(\omega), x_{n+1}(\omega)\right) \leq h\left(d\left(x_{n-1}(\omega), x_{n}(\omega)\right)\right)$

Similarly

$d\left(x_{n-1}(\omega), x_{n}(\omega)\right) \leq h\left(d\left(x_{n-2}(\omega), x_{n-1}(\omega)\right)\right.$

Hence $d\left(x_{n}(\omega), x_{n+1}(\omega)\right) \leq h\left(d\left(x_{n-1}(\omega), x_{n}(\omega)\right)\right) \leq$ $h^{2}\left(d\left(x_{n-2}(\omega), x_{n-1}(\omega)\right)\right)$

On continuing this process

$d\left(x_{n}(\omega), x_{n+1}(\omega)\right) \leq h^{n}\left(d\left(x_{0}(\omega), x_{1}(\omega)\right)\right)$

So for $n>m$

$d\left(x_{m}(\omega), x_{n}(\omega)\right) \leq\left(h^{m}+h^{m+1}+h^{m+2}+\cdots \ldots \ldots+\right.$

$\left.h^{n-1}\right)\left(d\left(x_{0}(\omega), x_{1}(\omega)\right)\right)$

$\leq \frac{h^{m}}{1-h}\left(d\left(x_{0}(\omega), x_{1}(\omega)\right)\right)$

Let $0 \ll c$ be given. Choose a natural number $N$ such that $\frac{h^{m}}{1-h}\left(d\left(x_{0}(\omega), x_{1}(\omega)\right)\right) \ll c$ for every $m \geq N$.Thus

$d\left(x_{m}(\omega), x_{n}(\omega)\right) \leq \frac{h^{m}}{1-h}\left(d\left(x_{0}(\omega), x_{1}(\omega)\right)\right) \ll c$ for every $n>m \geq N$.

Therefore the sequence $\left\{x_{n}(\omega)\right\}$ is a Cauchy sequence in $\Omega \times X$ such that $x_{n}(\omega) \rightarrow z(\omega)$.

Choose a natural number $N_{1}$ such that

Hence we have

$d(z(\omega), T z(\omega)) \leq d\left(z(\omega), x_{n+1}(\omega)\right)+$

$d\left(x_{n+1}(\omega), \operatorname{Tz}(\omega)\right)$

$=d\left(z(\omega), x_{n+1}(\omega)\right)+d\left(T x_{n}(\omega), T z(\omega)\right) \leq$

$d\left(z(\omega), x_{n+1}(\omega)\right)+$

$\emptyset\left(d\left(x_{n}(\omega), z(\omega)\right), d\left(z(\omega), T x_{n}(\omega)\right)+d(z(\omega), T z(\omega))\right.$,

$d\left(x_{n}(\omega), T x_{n}(\omega)\right)+d\left(z(\omega), T x_{n}(\omega)\right)$,

$d\left(x_{n}(\omega), T z(\omega)\right)+d\left(z(\omega), T x_{n}(\omega)\right)$

$\leq d\left(z(\omega), x_{n+1}(\omega)\right)+$

$\emptyset\left(d\left(x_{n}(\omega), z(\omega)\right), d\left(z(\omega), x_{n+1}(\omega)\right)+d(z(\omega), T z(\omega))\right.$,

$d\left(x_{n}(\omega), x_{n+1}(\omega)\right)+$

$d\left(z(\omega), x_{n+1}(\omega)\right), d\left(x_{n}(\omega), T z(\omega)\right)+d\left(z(\omega), x_{n+1}(\omega)\right)$

Taking $n \rightarrow \infty$ we have

$d(z(\omega), T z(\omega)) \leq 0+\emptyset(0, d(z(\omega), T z(\omega))+0,0+$

$0, d(z(\omega), T z(\omega))+0)$

$d(z(\omega), T z(\omega)) \leq \emptyset(0,0+d(z(\omega), T z(\omega)), 0+$

$0, d(z(\omega), T z(\omega))+0))$

$d(z(\omega), T z(\omega)) \leq 0$

Thus $-(d(z(\omega), T z(\omega))) \in P$. But $d(z(\omega), T z(\omega)) \in P$. Therefore $d(z(\omega), T z(\omega))=0$ and so $\operatorname{Tz}(\omega)=z(\omega)$. 
Example: Let $M=R$ and $P=\{x \in M: x \geq 0\}$, also $\Omega=$ $[0,1]$ and $\sum$ be the sigma algebra of Lebesgue's measurable subset of $[0,1]$. Let $X=[0, \infty)$ and define mapping as $d:(\Omega \times X \quad) \times(\Omega \times X) \rightarrow M \quad$ by $d(x(\omega), y(\omega))=$ $|x(\omega)-y(\omega)|$. Then $(X, d)$ is a cone random metric space. Define random operator $T$ from $\Omega \times X$ to $X$ as $T(x(\omega))=$ $x(\omega) / 2$. Also sequence of mapping $x_{n}: \Omega \rightarrow X$ is defined by $x_{n}(\omega)=\left\{\left(1-(\omega / 2)^{2}\right)^{1+1 / 2}\right\}$ for every $\omega \in \Omega$ and $n \in$ $N$. Defined measurable mapping $x: \Omega \rightarrow X$ as $x(\omega)=\{1-$ $\left.(\omega / 2)^{2}\right\}$ is fixed point of the space.

Theorem 3.2: Let $(X, d)$ be a complete cone random metric space and let $M$ be a nonempty separable closed subset of cone metric space $X$ and let $S$ and $T$ be continuous random operators defined on $M$ such that for $\omega \in \Omega, T(\omega,):. \Omega \times$ $M \rightarrow M$ satisfying contraction

$d\left(S^{r}\left(x(\omega), T^{r} y(\omega)\right)\right) \leq$

$\emptyset\left(d(x(\omega), y(\omega)),\left[d\left(x(\omega), S^{r}(x(\omega))+\right.\right.\right.$

$\left.\left.d\left(y(\omega), T^{r} y(\omega)\right)\right)\right]$

$d\left(x(\omega), T^{r} y(\omega)\right)+$

$\left.d\left(S^{r} x(\omega)\right), y(\omega)\right) \ldots \ldots \ldots \ldots \ldots \ldots(3.2(a))$

For all $x, y \in X, \omega \in \Omega$ and $r>0$. Then $S$ and $T$ has common fixed point in $X$.

Proof: For each $x_{0}(\omega) \in \Omega \times X$, let us choose $x_{1}(\omega)=$ $S^{r} x_{0}(\omega)$ and $x_{2}(\omega)=T^{r} x_{1}(\omega)$. In general $n \geq 1$, $x_{n+1}(\omega)=S^{r}\left(x_{n}(\omega)\right)$ and $x_{n+2}(\omega)=T^{r}\left(x_{n+1}(\omega)\right)$. Then $d\left(x_{n+1}(\omega), x_{n+2}(\omega)\right)=d\left(S^{r}\left(x_{n}(\omega)\right), T^{r}\left(x_{n+1}(\omega)\right)\right)$ $\leq \varnothing\left(d\left(\left(x_{n}(\omega),\left(x_{n+1}(\omega)\right),\left[d\left(\left(x_{n}(\omega), S^{r}\left(x_{n}(\omega)\right)+\right.\right.\right.\right.\right.\right.$ $d\left(x_{n+1}(\omega), T^{r}\left(x_{n+1}(\omega)\right)\right]$,

$\left[d\left(x_{n}(\omega), T^{r}\left(x_{n+1}(\omega)\right)+d\left(S^{r}\left(x_{n}(\omega), x_{n+1}(\omega)\right)\right]\right)\right.$ $\leq \emptyset\left(d\left(\left(x_{n}(\omega),\left(x_{n+1}(\omega)\right),\left[d\left(\left(x_{n}(\omega),\left(x_{n+1}(\omega)\right)+\right.\right.\right.\right.\right.\right.$ $d\left(\left(x_{n+1}(\omega),\left(x_{n+2}(\omega)\right)\right]\right.$,

$\left[d\left(\left(x_{n}(\omega),\left(x_{n+2}(\omega)\right)+d\left(\left(x_{n+1}(\omega),\left(x_{n+1}(\omega)\right)\right]\right)\right.\right.\right.$ $\leq \emptyset\left(d\left(\left(x_{n}(\omega),\left(x_{n+1}(\omega)\right),\left[d\left(\left(x_{n}(\omega),\left(x_{n+1}(\omega)\right)+\right.\right.\right.\right.\right.\right.$ $d\left(\left(x_{n+1}(\omega),\left(x_{n+2}(\omega)\right)\right]\right.$,

$\left[d\left(\left(x_{n}(\omega),\left(x_{n+1}(\omega)\right)\right)\right]\right)$

Therefore by definition (2.8) we have

$d\left(\left(x_{n+1}(\omega),\left(x_{n+2}(\omega)\right) \leq h\left(d\left(\left(x_{n}(\omega),\left(x_{n+1}(\omega)\right)\right)\right.\right.\right.\right.$

Similarly

$d\left(\left(x_{n-1}(\omega),\left(x_{n}(\omega)\right) \leq h\left(d\left(\left(x_{n-2}(\omega),\left(x_{n-1}(\omega)\right)\right.\right.\right.\right.\right.$

Hence $d\left(\left(x_{n}(\omega),\left(x_{n+1}(\omega)\right) \leq h\left(d\left(\left(x_{n-1}(\omega),\left(x_{n}(\omega)\right)\right) \leq\right.\right.\right.\right.$ $h^{2} d\left(\left(x_{n-2}(\omega),\left(x_{n-1}(\omega)\right)\right.\right.$

On continuing this process

$d\left(\left(x_{n}(\omega),\left(x_{n+1}(\omega)\right) \leq h^{n}\left(d\left(\left(x_{0}(\omega),\left(x_{1}(\omega)\right)\right)\right.\right.\right.\right.$

So for $n>m$

$d\left(\left(x_{m}(\omega),\left(x_{n}(\omega)\right) \leq\left(h^{m}+h^{m+1}+h^{m+2}\right.\right.\right.$ $+$

$\left.h^{n-1}\right)\left(d\left(\left(x_{0}(\omega),\left(x_{1}(\omega)\right)\right)\right.\right.$

$\leq \frac{h^{m}}{1-h}\left(d\left(x_{0}(\omega), x_{1}(\omega)\right)\right)$

Let $0 \ll c$ be given. Choose a natural number $N$ such that $\frac{h^{m}}{1-h}\left(d\left(x_{0}(\omega), x_{1}(\omega)\right)\right) \ll c$ for every $m \geq N$. Thus $d\left(\left(x_{m}(\omega),\left(x_{n}(\omega)\right) \leq \frac{h^{m}}{1-h}\left(d\left(x_{0}(\omega), x_{1}(\omega)\right)\right) \ll c\right.\right.$ for every $n>m \geq N$.

Therefore the sequence $\left\{x_{n}(\omega)\right\}$ is a Cauchy sequence in $\Omega \times X$. Since $(X, d)$ is complete, there exists $z(\omega) \in \Omega \times X$ such that $x_{n}(\omega) \rightarrow z(\omega)$. Choose a natural number $N_{1}$ such that

Hence, we have

$$
\begin{aligned}
& d(z(\omega), T z(\omega)) \leq d\left(z(\omega), x_{n+1}(\omega)\right)+ \\
& d\left(x_{n+1}(\omega), T z(\omega)\right) \\
& =d\left(z(\omega), x_{n+1}(\omega)\right)+d\left(S^{r}\left(x_{n}(\omega), T^{r} z(\omega)\right)\right. \\
& \leq d\left(z(\omega), x_{n+1}(\omega)\right)+ \\
& \varnothing\left(d\left(x_{n}(\omega), z(\omega)\right),\left[d\left(x_{n}(\omega), S^{r}\left(x_{n}(\omega)\right), z(\omega)\right)\right],\right. \\
& {\left[d\left(x_{n}(\omega), T^{r} z(\omega)\right)+d\left(S^{r}\left(x_{n}(\omega), z(\omega)\right)\right]\right)} \\
& \leq d\left(z(\omega), x_{n+1}(\omega)\right)+ \\
& \varnothing\left(d\left(x_{n}(\omega), z(\omega)\right),\left[d\left(z(\omega), x_{n+1}(\omega)\right)+\right.\right. \\
& \left.d\left(z(\omega), T^{r} z(\omega)\right)\right], \\
& \left.\left[d\left(x_{n}(\omega), T^{r} z(\omega)\right)+d\left(x_{n+1}(\omega), z(\omega)\right)\right]\right)
\end{aligned}
$$

Taking $n \rightarrow \infty$ we have

$d\left(z(\omega), T^{r} z(\omega)\right) \leq 0+\emptyset(0,[0+$

$\left.\left.d\left(z(\omega), T^{r} z(\omega)\right)\right],\left[d\left(z(\omega), T^{r} z(\omega)\right)+0\right]\right)$

$d\left(z(\omega), T^{r} z(\omega)\right) \leq$

$\emptyset\left(0, d\left(z(\omega), T^{r} z(\omega)\right), d\left(z(\omega), T^{r} z(\omega)\right)\right)$

$d\left(z(\omega), T^{r} z(\omega)\right) \leq 0$

Thus $-\left(d\left(z(\omega), T^{r} z(\omega)\right) \in P\right.$. But $d\left(z(\omega), T^{r} z(\omega)\right) \in P$.

Therefore $d\left(z(\omega), T^{r} z(\omega)\right)=0$ and so $T^{r} z(\omega)=z(\omega)$.

Similarly

$\left.\left.d\left(S^{r} z(\omega)\right), z(\omega)\right) \leq d\left(S^{r} z(\omega)\right), x_{n+2}(\omega)\right)+$

$d\left(x_{n+2}(\omega), z(\omega)\right)$

$\left.=d\left(S^{r} z(\omega)\right), T^{r} x_{n+1}(\omega)\right)+d\left(x_{n+2}(\omega), z(\omega)\right)$

$$
\begin{array}{r}
\leq \emptyset\left(d\left(z(\omega), x_{n+1}(\omega)\right),[d(z(\omega), S(z(\omega)))\right. \\
\left.+d\left(x_{n+1}(\omega), T^{r} x_{n+1}(\omega)\right)\right],
\end{array}
$$

$\left.\left[d\left(z(\omega), T^{r} x_{n+1}(\omega)\right)+d\left(S^{r} z(\omega)\right), x_{n+1}(\omega)\right)\right]+$

$d\left(x_{n+2}(\omega), z(\omega)\right)$

$\leq \varnothing\left(d\left(z(\omega), x_{n+1}(\omega)\right),\left[d\left(z(\omega), S^{r} z(\omega)\right)\right)+\right.$

$\left.d\left(x_{n+1}(\omega), x_{n+1}(\omega)\right)\right]$,

$\left[d\left(z(\omega), x_{n+2}(\omega)\right)+d\left(S\left(z(\omega), x_{n+1}(\omega)\right)\right]+\right.$

$d\left(x_{n+2}(\omega), z(\omega)\right)$

Taking $n \rightarrow \infty$ we have

$\left.d\left(S^{r} z(\omega)\right), z(\omega)\right) \leq$

$\varnothing\left(d(z(\omega), z(\omega)),\left[d(z(\omega), z(\omega)),\left[d\left(z(\omega), S^{r} z(\omega)\right)\right)+\right.\right.$

$d(z(\omega), z(\omega))]$,

$\left.\left[d(z(\omega), z(\omega))+d\left(S^{r} z(\omega)\right), z(\omega)\right)\right]+d(z(\omega), z(\omega))$ $\left.d\left(S^{r} z(\omega)\right), z(\omega)\right)$

$\leq \varnothing\left(0,\left[d\left(z(\omega), S^{r} z(\omega)\right)\right)+0\right],[0$

$\left.\left.+d\left(S^{r} z(\omega)\right), z(\omega)\right)\right]+0$

$\left.d\left(S^{r} z(\omega)\right), z(\omega)\right)$

$\left.\left.\leq \varnothing\left(0, d\left(z(\omega), S^{r} z(\omega)\right)\right), d\left(S^{r} z(\omega)\right), z(\omega)\right)\right)$

$\left.d\left(S^{r} z(\omega)\right), z(\omega)\right) \leq 0$

Thus - $\left.\left(d\left(S^{r} z(\omega)\right), z(\omega)\right)\right) \in P$. But $\left.d\left(S^{r} z(\omega)\right), z(\omega)\right) \in$ $P$.

Therefore $\left.d\left(S^{r} z(\omega)\right), z(\omega)\right)=0$ and so $S^{r}(z(\omega))=z(\omega)$. Hence $S^{r} z(\omega)=z(\omega)=T^{r}(z(\omega))$.

Theorem 3. 3: Let $(X, d)$ be a complete cone random metric space and let $M$ be a non-empty separable closed subset of cone metric space $X$ and let $T$ and $f$ be two continuous random operators defined on $M$. Assume that $T$ is a injective mapping and mapping $T$ and $f$ be such that for $\omega \in \Omega$, $T(\omega,):. \Omega \times M \rightarrow M$ satisfying contraction 
$d(\operatorname{Tx}(\omega), \operatorname{Tf}(y(\omega))) \leq$

$\varnothing(d(T x(\omega), T y(\omega)), d(T x(\omega), T f(x(\omega)))+$

$d(\operatorname{Tx}(\omega), T f(y(\omega)))$,

$d(\operatorname{Ty}(\omega), T f(y(\omega)))+$

$d(\operatorname{Tx}(\omega), T f(x(\omega))))$

For all $x, y \in X, \omega \in \Omega$, then $f$ has a unique fixed point in $X$. Moreover if $(T, f)$ is a Banach pair, then $T$ and $f$ have unique fixed point in $X$.

Proof: Let $x_{0}(\omega) \in \Omega \times X$ be arbitrary. Define a sequence $\left\{x_{n}\right\}$

$d\left(T x_{n}(\omega), T x_{n+1}(\omega)\right)=d\left(T f\left(x_{n-1}(\omega)\right), T f\left(x_{n}(\omega)\right)\right)$

$\leq \emptyset\left(d\left(T x_{n-1}(\omega), T x_{n}(\omega)\right), d\left(T x_{n-1}(\omega), T f\left(x_{n-1}(\omega)\right)\right)\right.$

$+d\left(T x_{n}(\omega), T f\left(x_{n}(\omega)\right)\right), d\left(T x_{n-1}(\omega), T f\left(x_{n}(\omega)\right)\right)$

$+d\left(T x_{n-1}(\omega), T f\left(x_{n-1}(\omega)\right)\right), d\left(T x_{n}(\omega), T f\left(x_{n}(\omega)\right)\right)$

$\left.+d\left(\operatorname{Tx} x_{n-1}(\omega), T f\left(x_{n-1}(\omega)\right)\right)\right)$

$=\varnothing\left(d\left(T x_{n-1}(\omega), T x_{n}(\omega)\right), d\left(T x_{n-1}(\omega), T x_{n}(\omega)\right)+\right.$

$d\left(T x_{n}(\omega), T x_{n+1}(\omega)\right)$,

$d\left(T x_{n-1}(\omega), T x_{n+1}(\omega)\right)+d\left(T x_{n-1}(\omega), T x_{n}(\omega)\right)$,

$\left.d\left(T x_{n}(\omega), T x_{n}(\omega)\right)+d\left(T x_{n-1}(\omega), T x_{n}(\omega)\right)\right)$

$=\varnothing\left(d\left(T x_{n-1}(\omega), T x_{n}(\omega)\right), d\left(T x_{n-1}(\omega), T x_{n}(\omega)\right)+\right.$

$d\left(T x_{n}(\omega), T x_{n+1}(\omega)\right)$,

$d\left(T x_{n-1}(\omega), T x_{n}(\omega)\right)+d\left(T x_{n-1}(\omega), T x_{n}(\omega)\right)$,

$\left.d\left(T x_{n}(\omega), T x_{n+1}(\omega)\right)+d\left(T x_{n-1}(\omega), T x_{n}(\omega)\right)\right)$

Hence we get,

$d\left(T x_{n}(\omega), T x_{n+1}(\omega)\right) \leq h d\left(T x_{n-1}(\omega), T x_{n}(\omega)\right)$

Similarly we can show that,

$d\left(T x_{n}(\omega), T x_{n+1}(\omega)\right) \leq h d\left(T x_{n-2}(\omega), T x_{n-1}(\omega)\right)$

In general we can write,

$d\left(T x_{n}(\omega), T x_{n+1}(\omega)\right) \leq h^{n} d\left(T x_{0}(\omega), T x_{1}(\omega)\right)$

So for $n>m$

$d\left(T x_{n}(\omega), T x_{m}(\omega)\right) \leq\left(h^{m}+h^{m+1}+h^{m+2}+\cdots \ldots \ldots \ldots+\right.$

$\left.h^{n-1}\right) d\left(T x_{0}(\omega), T x_{1}(\omega)\right)$

$\leq \frac{h^{m}}{1-h}\left(d\left(T x_{0}(\omega), T x_{1}(\omega)\right)\right) \ll a$ for every $m \geq N$.

Thus $d\left(T x_{n}(\omega), T x_{m}(\omega)\right) \leq \frac{h^{m}}{1-h}\left(d\left(T x_{0}(\omega), T x_{1}(\omega)\right)\right) \ll$ a $n>m \geq N$.

Therefore the sequence $\left\{x_{n}(\omega)\right\}$ is a Cauchy sequence in

$\Omega \times X$.

Since $(X, d)$ is complete, there exists $u(\omega) \in \Omega \times X$ such that

$T x_{n}(\omega) \rightarrow T u(\omega)$.

Since $T$ is subsequently convergent, $\left\{x_{n}(\omega)\right\}$ is such that $\lim _{n \rightarrow \infty} x_{n}(\omega)=z(\omega)$.

As $T$ is continuous $\lim _{n \rightarrow \infty} T x_{n}(\omega)=T z(\omega)$.

By uniqueness of limit $z(\omega)=T u(\omega)$

Since $f$ is continuous $\lim _{n \rightarrow \infty} f x_{n}(\omega)=f z(\omega)$.

Again as $T$ is continuous $\lim _{n \rightarrow \infty} T f x_{n}(\omega)=T f z(\omega)$

Therefore $\lim _{n \rightarrow \infty} T x_{n+1}(\omega) \stackrel{n \rightarrow \infty}{=} \operatorname{Tf} z(\omega)$

Choose a natural number $N_{1}$ such that for every $n \geq N$

$d\left(T z(\omega), T x_{n+1}(\omega)\right)=\ll \frac{a}{2}$

And $d\left(T x_{n}(\omega), T x_{n+1}(\omega)\right) \ll \frac{a}{2}$

Hence for $n \geq N_{1}$ we have

$$
\begin{aligned}
& d(T f(z(\omega)), T z(\omega)) \leq d\left(T f(z(\omega)), T x_{n+1}(\omega)\right)+ \\
& d\left(T x_{n+1}(\omega), T z(\omega)\right) \\
& =d\left(T f(z(\omega)), T f\left(x_{n}(\omega)\right)\right)+d\left(T x_{n+1}(\omega), T z(\omega)\right) \\
& \leq \emptyset\left(d\left(\operatorname{Tz}(\omega), T x_{n}(\omega)\right), d(\operatorname{Tz}(\omega), T f(z(\omega)))+\right. \\
& d\left(T x_{n}(\omega), T f\left(x_{n}(\omega)\right)\right) \\
& d\left(\operatorname{Tz}(\omega), T f\left(x_{n}(\omega)\right)\right)+ \\
& d(\operatorname{Tz}(\omega), T f(z(\omega))), d\left(T x_{n}(\omega), T f(z(\omega))\right) \\
& +d(\operatorname{Tz}(\omega), T f(z(\omega)))+d\left(T x_{n+1}(\omega), T z(\omega)\right) \\
& =\varnothing\left(d\left(\operatorname{Tz}(\omega), T x_{n}(\omega)\right), d(\operatorname{Tz}(\omega), T f(z(\omega)))+\right. \\
& d\left(T x_{n}(\omega), T f\left(x_{n+1}(\omega)\right)\right. \text {, } \\
& d\left(\operatorname{Tz}(\omega), T x_{n+1}(\omega)\right)+ \\
& d(\operatorname{Tz}(\omega), T f(z(\omega))), d\left(T x_{n}(\omega), T f(z(\omega))\right)+ \\
& d(\operatorname{Tz}(\omega), T f(z(\omega)))+d\left(T x_{n+1}(\omega), T z(\omega)\right) \\
& d(\operatorname{Tz}(\omega), \operatorname{Tf}(z(\omega))) \leq \\
& d\left(\operatorname{Tz}(\omega), T x_{n+1}(\omega)\right), d\left(T x_{n}(\omega), T f\left(x_{n+1}(\omega)\right)\right. \\
& \ll \frac{a}{2}+\frac{a}{2}=a \text { for every } n \geq N_{1} \\
& \text { Thus } d(\operatorname{Tz}(\omega), T f(z(\omega))) \ll \frac{a}{m} \text { for all } m \geq 1 \text {. } \\
& \text { So } \frac{a}{m}-d(\operatorname{Tz}(\omega), T f(z(\omega))) \in P \text { for all } m \geq 1 \text {. } \\
& \text { Since } \frac{a}{m} \rightarrow 0 \text { as } m \rightarrow \infty \text {, and } P \text { is closed } \\
& -d(\operatorname{Tz}(\omega), T f(z(\omega))) \in P \text {. But } d(\operatorname{Tz}(\omega), T f(z(\omega))) \in \\
& P \text {. } \\
& \text { Therefore } d(\operatorname{Tz}(\omega), T f(z(\omega)))=0 \\
& \text { And so } \operatorname{Tz}(\omega)=\operatorname{Tf}(z(\omega)) \text {. }
\end{aligned}
$$

As $T$ is injective $z(\omega)=f(z(\omega))$. Thus $z(\omega)$ is the fixed point of $f$.

Uniqueness: If $u(\omega)$ is another fixed point of $f$, then $u(\omega)=f(u(\omega))$.

$d(T u(\omega), T z(\omega))=d(T f(u(\omega)), T f(z(\omega)))$ $\leq \emptyset(d(T u(\omega), T z(\omega)), d(T u(\omega), T f(u(\omega))+$ $d(\operatorname{Tz}(\omega) \operatorname{Tf}(z(\omega)))$

$d(T u(\omega), T f(z(\omega))+d(T u(\omega), T f(u(\omega)))$,

$d(\operatorname{Tz}(\omega), T f(u(\omega))+d(T u(\omega), T f(u(\omega)))=$

$\emptyset(d(T u(\omega), T z(\omega)), d(T u(\omega), T u(\omega))+$

$d(\operatorname{Tz}(\omega), T z(\omega))$

$d(T u(\omega), T z(\omega))+d(T u(\omega), T u(\omega))$,

$d(T z(\omega), T u(\omega))+d(T u(\omega), T u(\omega))$

$=h d(T u(\omega), T z(\omega))$ as $h<1$, a contraction.

Hence $d(T u(\omega), T z(\omega))=0$ which implies $T u(\omega)=$ $T z(\omega)$

As $T$ is injective, $u(\omega)=z(\omega)$ is the unique fixed point of $f$.

As $(T, f)$ is a Banach pair, $T$ and $f$ commutes at fixed point of $f$ which implies that $T f z(\omega)=f T z(\omega)$ i.e. $\operatorname{Tz}(\omega)=$ $f T z(\omega)$ which implies that $\operatorname{Tz}(\omega)$ is another fixed point of $f$.

By uniqueness of fixed point of $f, z(\omega)=T z(\omega)$.

Hence $z(\omega)=f z(\omega)=T z(\omega)$ is the unique fixed point of $f$ and $T$ in $X$.

\section{REFERENCES}


[1] Agarwal, R.P. and O'Regan, D., Fixed point theory for generalized Contractions on spaces with two metrics, J. Math. Anal. Appl. 248 (2000), 402-414.

[2] Beg, I. and Azam, A., J. Austral. Math. Soc. Ser. A, 53 (1992) 313-26.

[3] Beg, I. and Shahzad, N., Nonlinear Anal., 20 (1993) 83547.

[4] Beg, I. and Shahzad, N., J. Appl. Math. and Stoch. Anal. 6 (1993) 95-106.

[5] Beg, I. and Shahzad, N., Random fixed points of weakly inward operators in conical shells, J. Appl. Math. Stoch. Anal. 8 (1995), 261-264.

[6] Beiranvand, A., Moradi, S., Omid M.and Pazandeh H., Two Fixed Point Theorems For Special Mappings, arXiv:0903.1504v1 math.FA, (2009).

[7] Berinde, V., Iterative approximation of fixed points, Lecture Notes in Mathematics, 1912,Springer, Berlin, 2007.

[8] Bharucha-Reid, A. T., "Random Integral Equations", Academic press, New York, 1972.

[9] Chen, J., Li, Z., Common Fixed Points For Banach Operator Pairs in Best Approximation, J.Math. Anal. Appl., 336(2007), 1466-1475.

[10] Dhagat, V.B., Sharma, A.K., Bharadwaj, R.K., Fixed point theorem for Random operators in Hilbert Spaces, International Journal of Mathema - tical Analysis 2 no. 12 (2008), 557-561.

[11] Hans, O., Reduzierende, Czech. Math. J. 7 (1957) 154-58.

[12] Hans, O., Random operator equations, Proc. $4^{\text {th }}$ Berkeley Symp. Math. Statist. Probability (1960), Vol. II, (1961) 180-202.

[13] Hardy, G. E. and Rogers, T. D., Canad. Math. Bull., 16 (1973) 201-206.

[14] Huang, L.G., Zhang, X., Cone metric spaces and a fixed point theorems of contractive mappings, J. Math. Anal. Appl. 332 (2007), 1468-1476.

[15] Itoh, S., Random fixed point theorems with an application to random differential equations in Banach spaces, J. Math. Anal. Appl. 67 (1979), 261-273.

[16] Kannan, R., Some results on fixed points. Bull.Calcutta Math.Soc., 10, 71-76 (1968).
[17] Pathak, H.K., Shahzad, N., Fixed point results for generalized quasi-contraction mappings in abstract metric spaces, Nonlinear Anal. 71 (2009), 6068- 6076.

[18] Kuratowski, K. and Ryll-Nardzewski, C., A general theorem on selectors, Bull. Acad. Polon. Sci. Ser. Sci. Math. Astronom. Phys. 13 (1965), 379-403.

[19] Lin, T.C., Random approximations and random fixed point theorems for continuous 1-set-contractive random maps, Proc. Amer. Math. Soc. 123 (1995), 1167-1176.

[20] Mehta Smrati, Singh A. D., Sanodia and Dhagat Vanita Ben, "Fixed point theorems for weak contraction in cone random metric spaces",Bull. Cal. Math. Soc.,103(4)pp 303-310,2011.

[21] O'Regan, D., A continuation type result for random operators, Proc. Amer. Math. Soc. 126 (1998), $1963-$ 1971.

[22] Papageorgiou, N.S., Random fixed point theorems for measurable Multi-functions in Banach spaces, Proc. Amer. Math. Soc. 97 (1986), 507-514.

[23] Rezapour, Sh., Hamlbarani, R., Some notes on the paper "Cone metric spaces and fixed points theorems of contractive mappings", J. Math. Anal. Appl. 345 (2008), 719-724.

[24] Rezapour, Sh., Haghi, R.H., Shahzad, N., Some notes on fixed points of quasi- contraction map, Applied Mathematics Letters, 23 (2010) 498-502.

[25] Rhoades, B. E., Sessa, S., Khan, M. S. and Swaleh, M., J. Austral. Math.Soc. ( Ser. A) 43 (1987) 328-46.

[26] Sehgal, V. M. and Singh, S. P. Proc. Amer. Math. Soc., 95(1985) 91-94.

[27] Shahzad, N. and Latif, S., Random fixed points for several classes of 1-ball -contractive and 1-set-contractive random maps, J. Math. Anal. Appl. 237 (1999), 83-92.

[28] Spacek, A. Zufallige Gleichungen, Czechoslovak Math. J. 5 (1955) 462-466.

[29] Sumitra, V.R., Uthariaraj,R. Hemavathy, Common Fixed Point Theorem For T- Hardy-Rogers Contraction Mapping in a Cone Metric Space, International Mathematical Forum5(2010), 1495-1506. 